\title{
Мощные оптоэлектронные коммутаторы нано- и пикосекундного диапазона на основе высоковольтных кремниевых структур с $p-n$-переходами. II. Энергетическая эффективность
}

\author{
(C) A.C. Кюрегян
}

Всероссийский электротехнический институт им. В.И. Ленина, 111250 Москва, Россия

E-mail: semlab@yandex.ru

(Получена 21 декабря 2016 г. Принята к печати 28 февраля 2017 г.)

Впервые изучена энергетическая эффективность оптоэлектронных коммутаторов на основе высоковольтных кремниевых фотодиодов, фототранзисторов и фототиристоров, управляемых пикосекундными лазерными импульсами, при формировании импульсов напряжения на активной нагрузке $R_{L}$. Показано, что при заданных значениях амплитуды $U_{R}$ и длительности $t_{R}$ импульсов существуют оптимальные величины площади приборов, энергии и коэффициента поглощения управляющего излучения, обеспечивающие максимальный общий коэффициент полезного действия коммутатора $\sim 0.92$. Все три типа коммутаторов обладают практически одинаковой эффективностью при малых $t_{R}$, а при больших $t_{R}$ заметным преимуществом обладают фототиристоры.

DOI: $10.21883 / F T P .2017 .09 .44892 .8495$

В работе автора [1] были изложены результаты численного моделирования переключения высоковольтных кремниевых структур с $p-n$-переходами, управляемых пикосекундными лазерными импульсами, и получены соотношения между параметрами коммутаторов и характеристиками процесса переключения. Настоящая статья является продолжением [1], однако теперь основное внимание будет сосредоточено на анализе не изученного ранее вопроса об энергетической эффективности оптоэлектронных коммутаторов, которая фактически и определяет целесообразность практического применения этих приборов.

Все обозначения, а также объекты и метод исследования остались теми же, как и значения основных параметров коммутаторов: $U_{0}=5 \kappa \mathrm{B}, t_{\mathrm{ph}}=10$ пс, $R_{L}=5 \mathrm{Oм}$, $T=75^{\circ} \mathrm{C}$ и, если это особо не оговорено, $S_{\mathrm{ph}}=0.5 \mathrm{~cm}^{2}$, $\kappa=32 \mathrm{~cm}^{-1}$.

Энергетическая эффективность коммутаторов характеризуется общим коэффициентом полезного действия (кпд)

$$
\eta_{\mathrm{tot}}=\frac{W_{R}}{W_{R}+W_{\mathrm{tot}}}
$$

где $W_{R}$ - энергия, рассеянная сопротивлением нагрузки $R_{L}, W_{\text {tot }}=W_{D}+W_{\mathrm{ph}} / \eta_{\text {las }}$ - общая энергия потерь коммутатора, $W_{D}$ - энергия, рассеянная переключающим прибором, $W_{\mathrm{ph}}$ - энергия управляющего импульса света, $\eta_{\text {las }}$ - кпд лазера „от розетки“, который мы полагали равным 0.1 [2]. Все эти энергии зависят от времени, как изображено на рис. 1. Поэтому кпд коммутатора должен зависеть от длительности импульса $t_{R}$.

На первом этапе процесса коммутации емкость структуры $C_{D}$ разряжается током проводимости. Энергия, рассеиваемая за это время нагрузкой, пренебрежимо мала, поэтому практически вся энергия $W_{C}=C_{D} U_{0}^{2} / 2$ $\approx 0.24$ мДж, накопленная в барьерной емкости прибора, рассеивается им самим.
На втором этапе мощность, рассеиваемая фотодиодом, пренебрежимо мала, так что энергия $W_{D}$ остается практически постоянной и равной $W_{C}$, a $W_{R}$ линейно увеличивается со временем вплоть до начала формирования ОПЗ в фотодиоде в момент $t=t_{s c}$. Далее напряжение на фотодиоде и, следовательно, энергия $W_{D}$ увеличиваются значительно быстрее $W_{R}$. Все это приводит к немонотонным зависимостям $\eta_{\text {tot }}$ от $t_{R}$, примеры которых изображены на рис. 2. Видно, что функция $\eta_{\mathrm{tot}}\left(t_{R}\right)$ достигает своего максимума $\eta_{\max }$ при некотором значении $t_{R}=t_{\max }$.

C ростом $W_{\text {ph }}$ максимальный кпд $\eta_{\max }$ сначала быстро увеличивается, но при больших $W_{\mathrm{ph}}$ стремится к постоянной величине $\sim 0.95$ (см. рис. 3 ). В то же время энергия $W_{R \max }=W_{R}\left(t_{\max }\right)$, рассеиваемая нагрузкой за

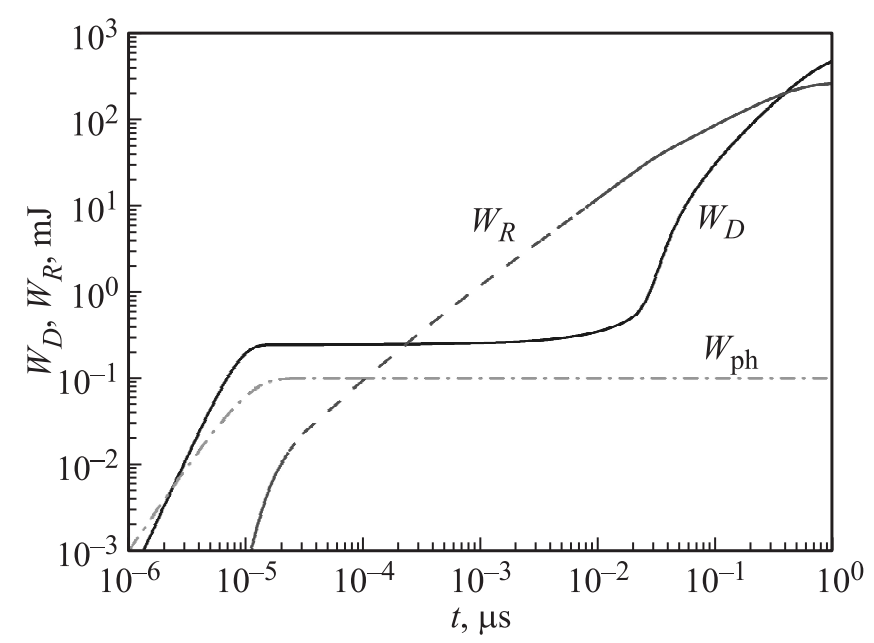

Рис. 1. Зависимости энергий, рассеиваемых нагрузкой $\left(W_{R}\right)$ и фотодиодом $\left(W_{D}\right)$, от времени $t$ при амплитуде импульса света $W_{\mathrm{ph}}=100$ мкДж. 


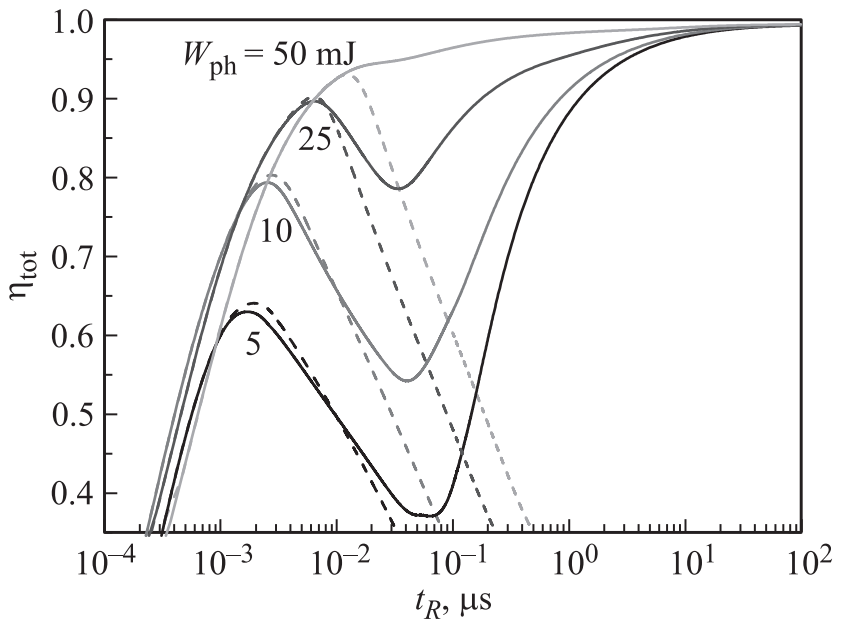

Pис. 2. Зависимость общего кпд $\eta_{\text {tot }}$ фотодиодного (штриховые линии) и фототиристорного (сплошные линии) коммутаторов от длительности импульса тока через нагрузку $t_{R}$ при $\eta_{\text {las }}=0.1$ и различных значениях $W_{\text {ph }}$.

время $t_{\max }$, и само время $t_{\max }$ линейно увеличиваются с ростом $W_{\text {ph }}$, в частности

$$
W_{R \max } \approx 300 W_{\mathrm{ph}}
$$

Практически такие же зависимости $W_{R \max }, t_{\max }$ и $\eta_{\max }$ от $W_{\text {ph }}$ получились для фототранзисторов во всем диапазоне значений $W_{\mathrm{ph}}$, а для фототиристоров только при $W_{\mathrm{ph}} \leq 35$ мкДж, когда еще существует локальный максимум функции $\eta_{\text {tot }}\left(t_{R}\right)$. Величины $\eta_{\max }$ и $t_{\max }$ зависят также от площади приборов и от коэффициента поглощения света, как это изображено на рис. 4 и 5.

Из рис. 3-5 следует, что во всех рассмотренных случаях $t_{\max }<t_{\mathrm{av}}$. Это означает, что падение кпд ограничивает допустимую длительность импульса, формируемого фотодиодным коммутатором, сильнее, чем наступление опасного динамического лавинного пробоя.

Если длительность $t_{R}$ импульса напряжения считать априори заданной величиной наряду с амплитудой $U_{R} \approx U_{0} / 2$ и $R_{L}$, то остается три свободных параметра коммутатора: $W_{\mathrm{ph}}, S_{\mathrm{ph}}$ и $\kappa$. Зависимости $\eta_{\text {tot }}$ и $W_{D}$ от этих параметров изображены на рис. 6-8 для $t_{R}=10$ нс. Видно, что существуют оптимальные значения $W_{\mathrm{ph}} \approx 50$ мкДж, $S_{\mathrm{ph}} \approx 0.5 \mathrm{~cm}^{2}$ и $\kappa \approx 32 \mathrm{~cm}^{-1}$, при которых кпд $\eta_{\text {tot }}$ максимален, а энергия $W_{D}$ и/или $W_{\text {tot }}$ минимальна. Причина существования минимума функции $W_{\text {tot }}\left(W_{\text {ph }}\right)$ очевидна - см. рис. 6.

C ростом $S_{\mathrm{ph}}$ уменьшается плотность тока, поэтому увеличивается время $t_{s c}$ возникновения ОПЗ (см. рис. 7 в [1]) и, следовательно, снижаются потери на срезе импульса. В то же время потери на фронте, равные $W_{C}$, пропорциональны $S_{\mathrm{ph}}$, что и приводит к возникновению минимума функции $W_{\text {tot }}\left(S_{\mathrm{ph}}\right)$.

Наконец, минимум функции $W_{D}(\kappa)$, изображенной на рис. 8 , существует потому, что при малых $\kappa$ концентрация неравновесных носителей заряда мала во всей

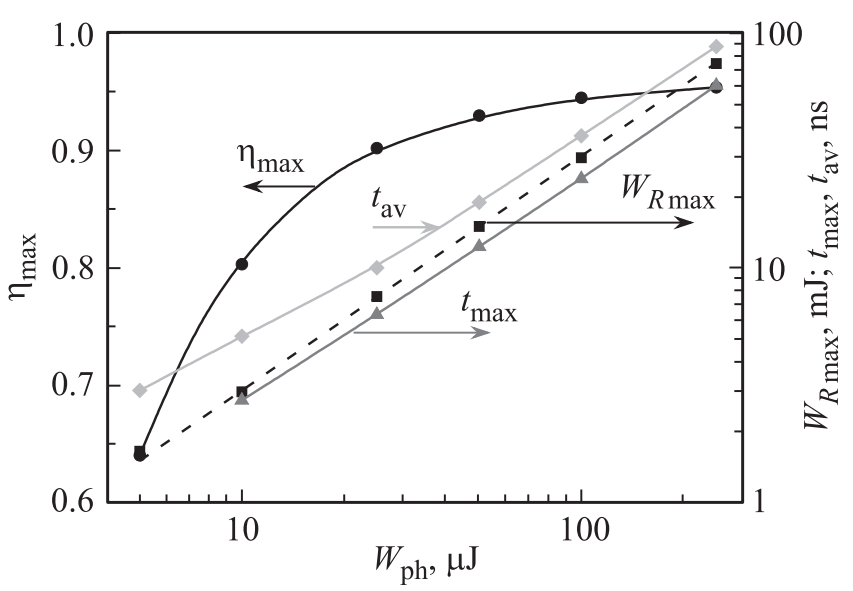

Рис. 3. Зависимости максимального полного кпд $\eta_{\max }$ фотодиодного коммутатора, энергии $W_{R \max }$, рассеиваемой нагрузкой за время $t_{\max }$, и времен $t_{\max }, t_{\mathrm{av}}$ от $W_{\mathrm{ph}}$ при $\eta_{\text {las }}=0.1$. Штриховая линия - расчет по формуле (2).

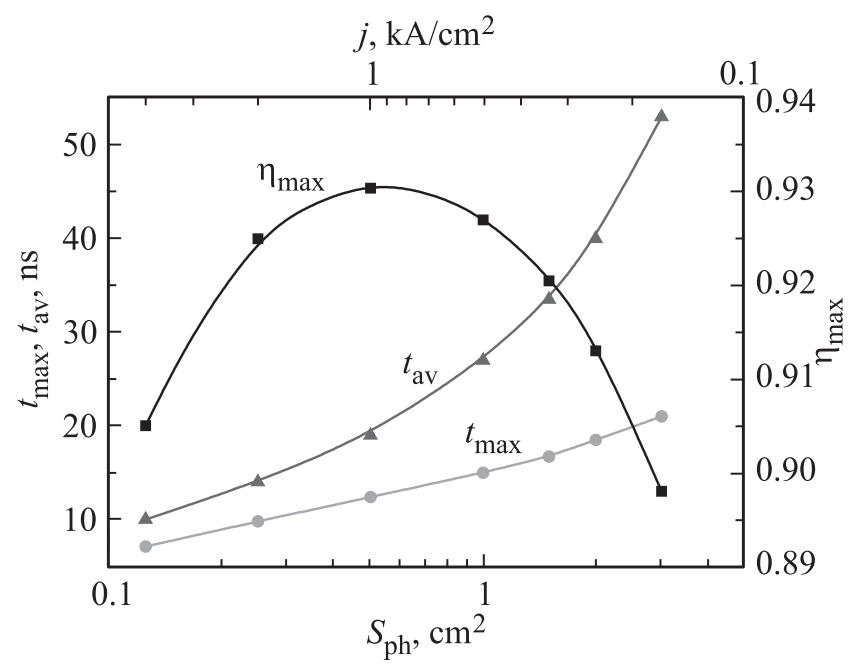

Рис. 4. Зависимости максимального полного кпд $\eta_{\max }$ фотодиодного коммутатора и времен $t_{\max }, t_{\mathrm{av}}$ от $S_{\mathrm{ph}}$ при $W_{\mathrm{ph}}=50$ мкДж.

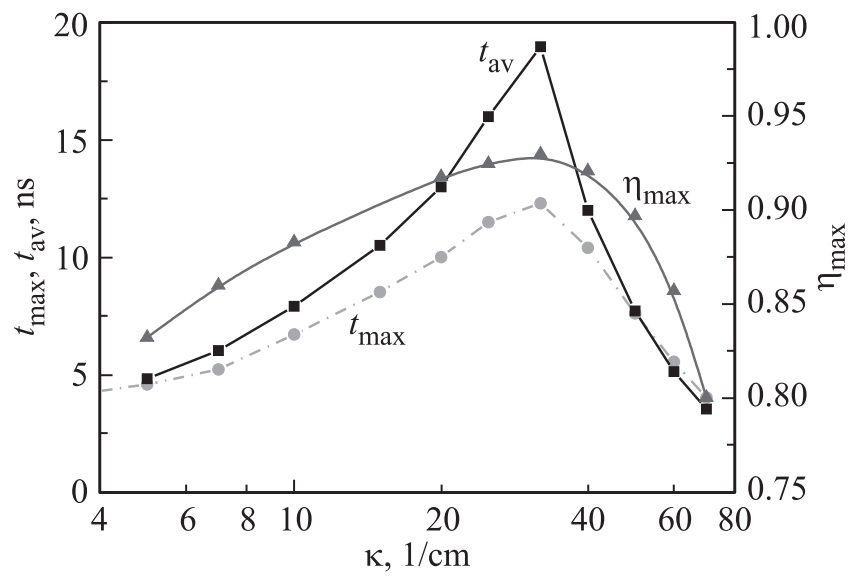

Рис. 5. Зависимости максимального полного кпд $\eta_{\max }$ фотодиодного коммутатора и времен $t_{\max }, t_{\mathrm{av}}$ от коэффициента поглощения $\kappa$ для фотодиода при $W_{\mathrm{ph}}=50$ мкДж. 


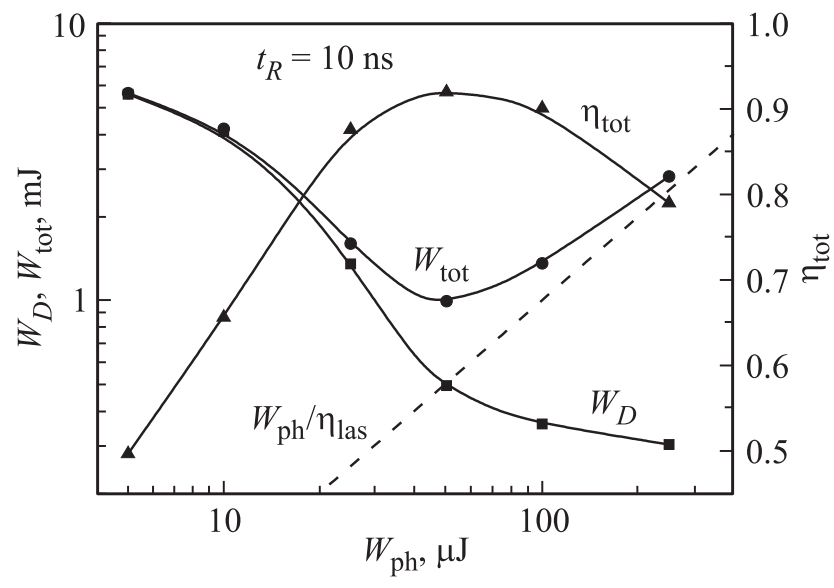

Рис. 6. Зависимости энергий $W_{D}, W_{\text {tot }}$ и общего кпд фотодиодного коммутатора $\eta_{\text {tot }}$ от $W_{\text {ph }}$ при длительности импульса тока нагрузки $t_{R}=10 \mathrm{Hc}$.

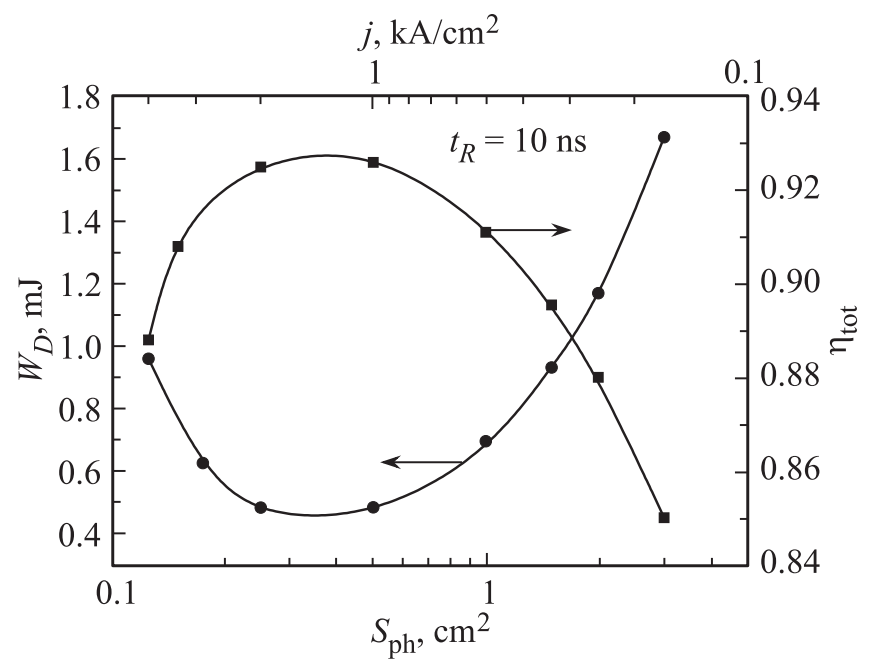

Рис. 7. Зависимости энергии $W_{D}$ и общего кпд фотодиодного коммутатора $\eta_{\text {tot }}$ от $S_{\mathrm{ph}}$ при длительности импульса тока нагрузки $t_{R}=10 \mathrm{Hc}$.

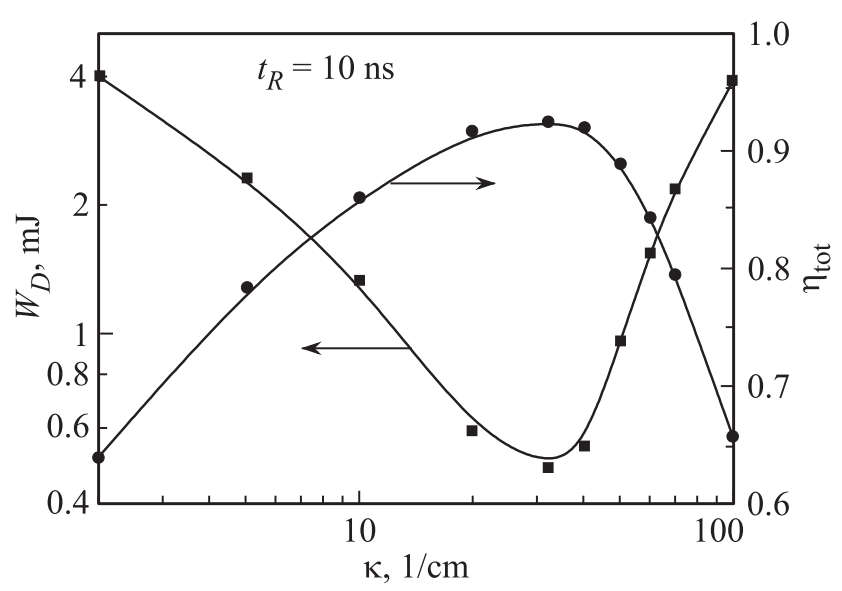

Рис. 8. Зависимости энергии $W_{D}$ и общего кпд коммутаторов $\eta_{\text {tot }}$ от $\kappa$ при длительности импульса тока нагрузки $t_{R}=10 \mathrm{Hc}$.

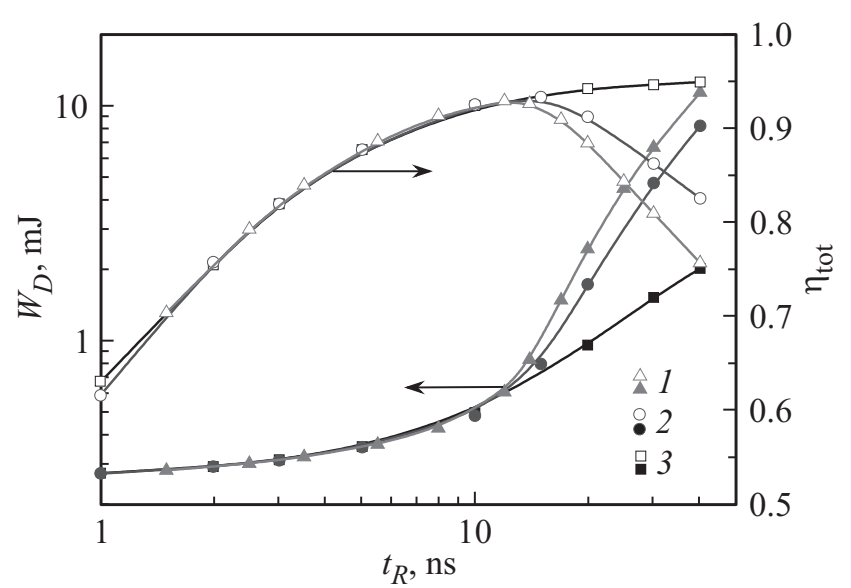

Рис. 9. Зависимости энергии $W_{D}$ и общего кпд $\eta_{\text {tot }}$ фотодиодных (1), фототранзисторных (2) и фототиристорных (3) коммутаторов от длительности импульса $t_{R}$ при $W_{\mathrm{ph}}=50$ мкДж.

структуре, а при больших - в прианодной области [1], где начинается „преждевременное“ формирование ОПЗ и рост потерь на срезе импульса.

Приведенные на рис. 3-8 результаты моделирования относятся к фотодиодным коммутатором. Однако, как было показано в [1], характеристики процессов переключения всех трех приборов практически идентичны до тех пор, пока в момент $t_{s c}$ не начинают формироваться области пространственного заряда. Только после этого начинает проявляться обычная инжекция одного (в фототранзисторах) или обоих (в фототиристорах) типов носителей заряда, отсутствующая в фотодиодах. Естественно, что и энергетические характеристики всех трех приборов должны совпадать при $t_{R}<t_{s c}$. Анализ результатов моделирования показывает, что они совпадают даже вплоть до $t_{R} \approx t_{\max } \approx(1.5-2) t_{s c}$. Например, при оптимальных значениях $W_{\mathrm{ph}} \approx 50$ мкДж, $S_{\mathrm{ph}} \approx 0.5 \mathrm{~cm}^{2}$ и $\kappa \approx 32 \mathrm{~cm}^{-1}$ преимущества фототранзисторов и особенно фототиристоров начинают проявляться только при $t_{R}>t_{\max } \approx 12.5$ нс (см. рис. 9).

Полученные результаты указывают на то, что использование оптопары на основе волоконного лазера и высоковольтной кремниевой структуры позволит создать почти идеальный коммутатор, способный формировать многокиловольтовые импульсы напряжения с фронтом менее 100 пс, длительностью до 10 нс и частотой повторения десятки килогерц при общем кпд „от розетки“ более 0.92 . По совокупности этих параметров такие приборы должны значительно превосходить все известные автору коммутаторы нано- и пикосекундного диапазона.

Автор благодарен С.Н. Юркову и Т.Т. Мнацаканову, предоставившим возможность провести моделирование процесса комммутации с помощью программы „Исследование“, и А.В. Горбатюку за интерес к работе и полезные обсуждения.

Работа выполнена при поддержке РФФИ (грант № 16-08-01292). 


\section{Список литературы}

[1] А.С. Кюрегян. ФТП, 51, 1. (2017).

[2] http://www.ipgphotonics.com/category/4/Lasers

Редактор Л.В. Шаронова

Power nano- and picosecond

optoelectronic switches based

on high-voltage silicon structures

with $p-n$ junctions.

II. Energy efficiency

\section{A.S. Kyuregyan}

All-Russian Electrical Engineering Institute, 111250 Moscow, Russia

Abstract Energy efficiency of optoelectronic switches based on high-voltage silicon photodiodes, phototransistors or photothyristors and triggered-on by picosecond laser pulses has been studied for the first time. For given values of resistive load, amplitude and duration $t_{R}$ of voltage pulses it is shown that there exist optimal sets of values for the device area, energy and absorption coefficient for triggering illumination, which provide the maximal general efficiency of the switch about 0.92. All three types of switches have almost identical efficiencies at short $t_{R}$, and in case of long $t_{R}$ photothyristors possess a noticeable advantage among them. 